\title{
Self-energy-corrected Dirac wave functions for advanced QED calculations in highly charged ions
}

\author{
Natalia S. Oreshkina $\odot,{ }^{1, *}$ Halil Cakir $\odot,{ }^{1}$ Bastian Sikora, ${ }^{1}$ Vladimir A. Yerokhin $\odot,{ }^{1,2}$ Vincent Debierre $\odot,{ }^{1}$ Zoltán Harman, \\ and Christoph H. Keitel ${ }^{1}$ \\ ${ }^{1}$ Max-Planck-Institut für Kernphysik, Saupfercheckweg 1, 69117 Heidelberg, Germany \\ ${ }^{2}$ Center for Advanced Studies, Peter the Great Saint Petersburg Polytechnic University, 195251 Saint Petersburg, Russia
}

(Received 6 December 2019; accepted 22 January 2020; published 24 March 2020)

\begin{abstract}
The procedure for the calculation of the self-energy-corrected wave function of the bound electron in the field of the nucleus is discussed. We present the related formulas and discuss the numerical difficulties and the methods used to overcome them. The results of the calculation are presented for a wide range of ions. Possible applications of the numerically obtained wave functions are discussed.
\end{abstract}

DOI: 10.1103/PhysRevA.101.032511

\section{INTRODUCTION}

Highly charged ions are commonly studied in research on fundamental physical systems. They can be used for highprecision tests of quantum electrodynamics (QED) [1-5], for the precise determination of fundamental constants [6-10], and in the search for the possible variation of the latter [11-13]. For these high-precision tests, very accurate experimental and theoretical results are both needed. In particular, for accurate calculations of the bound-electron $g$ factor, QED corrections should be included not only in the first order [so-called self-energy (SE) and vacuum-polarization (VP) corrections], but also beyond the first order. The leading contribution of the VP correction can be described in the Uehling approximation by a relatively simple short-range potential, allowing one to estimate two-loop diagrams containing VP loops in a straightforward way. The SE cannot be well represented by a local potential and thus has to be calculated rigorously, and therefore the corresponding corrections have be calculated straightforwardly, which is a more difficult problem.

In the current paper, we describe the procedure for the numerical calculation of the SE-corrected bound-state wave function (in the following SE-WF) in detail. Similar calculations were presented in Ref. [14] for heavy hydrogenlike ions. Here, we report on SE-WF calculations for the ground $1 s$ state of hydrogenlike ions in a wide range of nuclear charges $Z=$ 2-92. The tabulated SE-WFs, given in both coordinate and momentum representations, can be used for the calculations of numerous QED corrections. Specifically, these include, e.g., the irreducible part of the first-order QED corrections to the hyperfine splitting, $g$ factor, transition probabilities, and other

\footnotetext{
*Natalia.Oreshkina@mpi-hd.mpg.de

Published by the American Physical Society under the terms of the Creative Commons Attribution 4.0 International license. Further distribution of this work must maintain attribution to the author(s) and the published article's title, journal citation, and DOI. Open access publication funded by the Max Planck Society.
}

physical quantities [see Fig. 1(a)]; several two-loop QED corrections to the binding energy (the so-called Lamb shift) [see Fig. 1(b)]; and, finally, some of the two-loop QED corrections to the hyperfine splitting, $g$ factor, etc. [see Fig. 1(c)].

The paper is organized as follows. In Sec. II, we present the analytical formulas for the different contributions to the SE-WF, and in Sec. III we discuss the numerical evaluation of these contributions and connected problems. In Sec. IV we present our calculated SE-WFs and discuss their current numerical uncertainties. Also, our SE-WFs were used for the calculation of some SE corrections, presented already in the literature, such as the irreducible part of the SE correction to the $g$ factor and the loop-after-loop (LAL) SE contribution to the Lamb shift. Our results for these corrections and comparison with earlier works [15-17] can be found in Sec. V.

The relativistic system of units ( $\hbar=m=c=1)$ and Heaviside charge units $\left(\alpha=e^{2} /(4 \pi)\right)$ are used throughout the paper. Bold letters are used for 3 -vectors, the components of 3 -vectors are listed with Latin indices, whereas Greek letters denote 4-vector indices.

\section{SELF-ENERGY WAVE-FUNCTION CONTRIBUTIONS FOR SECOND-ORDER QED CORRECTIONS}

The SE correction for the bound electron in highly charged hydrogenlike ions was calculated for the first time in Ref. [18] by the method proposed in Ref. [19]. Precision calculations were performed in Refs. [20,21], and later improved [22,23]. The calculation procedure is described in detail in Refs. [24,25], and successfully used in later works [26-29]. The same method is also used in the present paper for the calculation of the SE-WF for the ground state of hydrogenlike ions.

Let us start with the definition of the unperturbed wave function of an electron in the field of the nucleus. For the state $a$ with the energy $\varepsilon_{a}$, the wave functions are determined as solutions of the Dirac equation with a spherically symmetric nuclear potential as [30]

$$
\psi_{a}(\mathbf{r})=\left(\begin{array}{c}
g_{n_{a} \kappa_{a}}(r) \Omega_{\kappa_{a} m_{a}}(\widehat{\mathbf{r}}) \\
i f_{n_{a} \kappa_{a}}(r) \Omega_{-\kappa_{a} m_{a}}(\widehat{\mathbf{r}})
\end{array}\right) .
$$


(a)

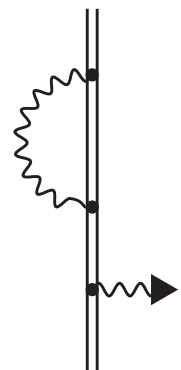

(b)

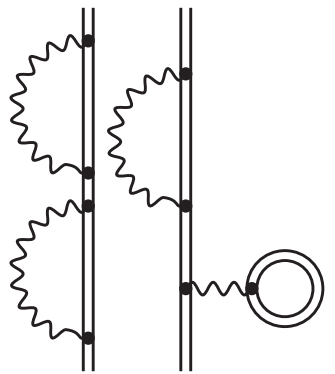

(c)

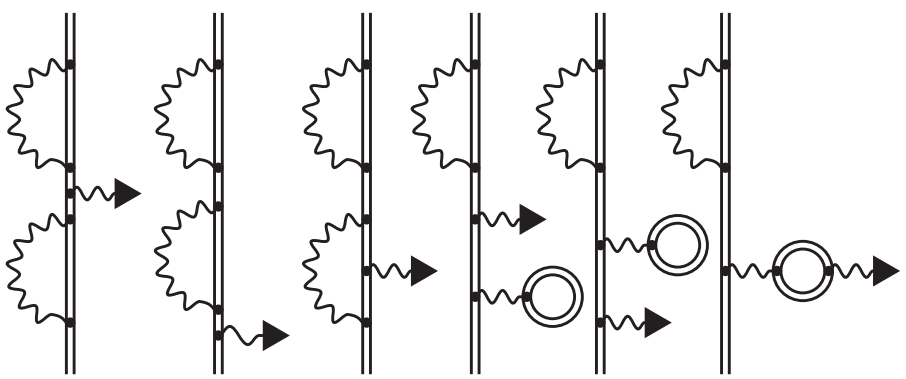

FIG. 1. Examples of QED corrections which can be calculated with the SE-WFs. (a) The self-energy loop is combined with an interaction with an arbitrary external field. (b) Two different two-loop QED-corrections diagrams, containing a self-energy loop, to the energy levels. (c) Diagrams for the two-loop QED corrections to the external field interaction of a bound electron, containing a self-energy loop. The SE loop here, and in the following figures, can be assumed to represent the renormalized SE operator $\Sigma_{R}$. Double lines represent bound-electron wave functions or propagators; the wavy line represents a virtual photon. A wavy line terminated by a triangle represents the interaction with an arbitrary external field.

Here, $n$ is the principal quantum number; $\kappa$ is the relativistic angular quantum number, which specifies both the total angular momentum quantum number $j=|\kappa|-1 / 2$ and the orbital angular momentum quantum number $l=|\kappa+1 / 2|-1 / 2 ; m$ is the projection of the total angular momentum. $\Omega_{\kappa m}(\widehat{\mathbf{r}})$ are the spherical spinors which are expressed as functions of $\widehat{\mathbf{r}} \equiv \mathbf{r} /|\mathbf{r}|$, and the radial components are normalized as

$$
\int_{0}^{\infty} d r r^{2}\left[g^{2}(r)+f^{2}(r)\right]=1 .
$$

The self-energy operator $\Sigma(E)$ can be written in terms of its matrix elements in the Feynman gauge as (see, e.g., Ref. [29])

$$
\begin{array}{r}
\langle a|\Sigma(E)| b\rangle=\frac{i}{2 \pi} \int_{-\infty}^{\infty} d \omega \sum_{n} \frac{\langle a n|I(\omega)| n b\rangle}{E-\omega-\varepsilon_{n}(1-i 0)}, \\
I\left(\omega, \mathbf{x}_{1}, \mathbf{x}_{2}\right)=\alpha \frac{\left(1-\boldsymbol{\alpha}_{1} \boldsymbol{\alpha}_{2}\right) \exp \left(i \sqrt{\omega^{2}+i 0} x_{12}\right)}{x_{12}} .
\end{array}
$$

Here, $x_{12}$ is the relative distance $x_{12}=\left|\mathbf{x}_{1}-\mathbf{x}_{2}\right|$, and the summation in Eq. (2) goes over the full spectrum of the Dirac equation, including negative- and positive-energy states. This expression suffers from ultraviolet divergences, and has to be considered together with a mass counterterm (see Fig. 2):

$$
\Sigma(E) \rightarrow \Sigma_{R}(E)=\Sigma(E)-\gamma^{0} \delta m .
$$

Here and below $\boldsymbol{\alpha}_{i}$ and $\gamma^{\mu}$ are Dirac matrices, $\delta m$ originates from the counterterm diagram, and $\Sigma_{R}$ stands for the renormalized SE operator.

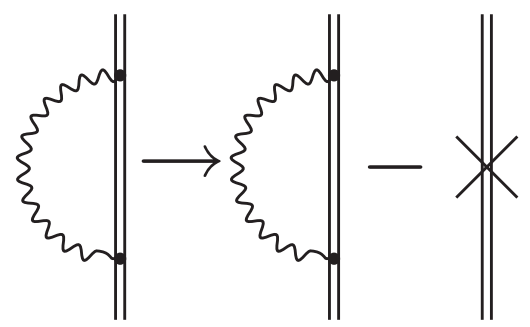

FIG. 2. Furry-picture Feynman diagram representing the renormalization of the self-energy diagram by the counterterm diagram. The cross indicates the mass counterterm.
With the notations introduced above, one can write the SEWF for the state $a$ as

$$
\left|\delta_{\mathrm{SE}} a\right\rangle=\sum_{n, n \neq a} \frac{|n\rangle\left\langle n\left|\Sigma_{R}\left(\varepsilon_{a}\right)\right| a\right\rangle}{\varepsilon_{a}-\varepsilon_{n}} \equiv \sum_{n}^{\prime} \frac{|n\rangle \Delta E_{n a}}{\varepsilon_{a}-\varepsilon_{n}} .
$$

This expression has the form of the standard quantummechanical first-order perturbation of the wave function $|a\rangle$ by the self-energy operator $\Sigma\left(\varepsilon_{a}\right)$ and thus is termed the SE-WF. We stress that the SE-WF delivers only a part of the total contribution induced by the electron self-energy. Corrections beyond the SE-WF arising within a rigorous QED perturbation theory should be taken into account separately within different approaches, which are beyond the scope of the present paper. In particular, this stands for the term with $n=a$, excluded from the summation in Eq. (4), which leads to the so-called reducible contributions. Please note that the SE operator is diagonal in the relativistic angular quantum number $\kappa$ and the angular momentum projection $m$, therefore the summation over $n$ in Eq. (4) goes only over the principal quantum number. Therefore, also, the spin-angular structure of the SE-WF is the same as that of the unperturbed Dirac wave function given in Eq. (1):

$$
\left|\delta_{\mathrm{SE}} a\right\rangle=\left(\begin{array}{c}
\delta_{\mathrm{SE}} g_{n_{a} \kappa_{a}}(r) \Omega_{\kappa_{a} m_{a}}(\widehat{\mathbf{r}}) \\
i \delta_{\mathrm{SE}} f_{n_{a} \kappa_{a}}(r) \Omega_{-\kappa_{a} m_{a}}(\widehat{\mathbf{r}})
\end{array}\right) .
$$

In the following, even if it is not indicated explicitly, we imply that $\Delta E_{n a} \propto \delta_{\kappa_{a}, \kappa_{n}} \delta_{m_{a}, m_{n}}$.

Let us discuss how the SE-WFs, determined by Eq. (4), can be used for practical calculations. As a first example, we consider the diagram in Fig. 1(a). If a triangle on this diagram corresponds to the interaction with an external magnetic field $\mathbf{B}$, the diagram describes the irreducible part of the SE correction to the bound-electron $g$ factor. The corresponding energy shift can be written as

$$
\Delta E_{\mathrm{SE}, \text { irred }}=2 \sum_{n}^{\prime} \frac{\langle a|(-e \boldsymbol{\alpha} \cdot \mathbf{A})| n\rangle\left\langle n\left|\Sigma_{R}\left(\varepsilon_{a}\right)\right| a\right\rangle}{\varepsilon_{a}-\varepsilon_{n}},
$$

where $e<0$ is the electron charge, $\mathbf{A}$ is the vector potential corresponding to the magnetic field $\mathbf{B}$, and the factor of 2 is due to the fact that there are two equivalent diagrams. The usual approach to calculate this contribution is based on 
introducing the magnetic-field corrected WF

$$
\left|\delta_{\mathrm{B}} a\right\rangle=\sum_{n}^{\prime} \frac{|n\rangle\langle n|(-e \boldsymbol{\alpha} \cdot \mathbf{A})| a\rangle}{\varepsilon_{a}-\varepsilon_{n}},
$$

and expressing the irreducible SE correction as a nondiagonal element of the SE operator, namely,

$$
\Delta E_{\mathrm{SE}, \text { irred }}=2\left\langle\delta_{\mathrm{B}} a\left|\Sigma_{R}\left(\varepsilon_{a}\right)\right| a\right\rangle .
$$

However, this contribution can be also calculated with the use of the SE-WF (4) as

$$
\Delta E_{\mathrm{SE}, \text { irred }}=2\left\langle a|(-e \boldsymbol{\alpha} \cdot \mathbf{A})| \delta_{\mathrm{SE}} a\right\rangle .
$$

As a second example, let us consider the loop-after-loop SE contribution to the Lamb shift, given by the first diagram in Fig. 1(b). Following the Feynman rules, the energy shift is determined by

$$
\Delta E_{\mathrm{LAL}}=\sum_{n}^{\prime} \frac{\left\langle a\left|\Sigma_{R}\left(\varepsilon_{a}\right)\right| n\right\rangle\left\langle n\left|\Sigma_{R}\left(\varepsilon_{a}\right)\right| a\right\rangle}{\varepsilon_{a}-\varepsilon_{n}},
$$

or, using the SE-WF (4), as

$$
\Delta E_{\mathrm{LAL}}=\left\langle a\left|\Sigma_{R}\left(\varepsilon_{a}\right)\right| \delta_{\mathrm{SE}} a\right\rangle .
$$

Calculations for the SE correction to the $g$ factor $[10,31,32]$ and hyperfine splitting [13,33,34] [Fig. 1(a)], as well as for the two-loop QED corrections to the energy levels [16,17,35-38] [Fig. 1(b)], were performed in the past. In our current paper, we used the results of these calculations as a consistency check for our SE-WFs (see Sec. V). However, our main aim is to use the SE-WFs for calculations of the SE-SE loopafter-loop and the irreducible part of the SE magnetic loop two-loop QED corrections to the $g$ factor, corresponding to the diagrams in Fig. 1(c). Some of these diagrams were already calculated with our SE-WFs, and the results are presented in Refs. [39,40].

As we can see from Eq. (4), the SE-WF can be written as a sum of renormalized nondiagonal elements of the SE operator. Therefore, the ultraviolet divergence can be treated with the standard dimensional regularization approach [29]. To separate this divergence, let us expand the Green's function for the bound electron in powers of the potential:

$$
\begin{aligned}
\frac{1}{\omega-H}= & \frac{1}{\omega-H_{0}}+\frac{1}{\omega-H_{0}} V \frac{1}{\omega-H_{0}} \\
& +\frac{1}{\omega-H_{0}} V \frac{1}{\omega-H} V \frac{1}{\omega-H_{0}} .
\end{aligned}
$$

Here, $H_{0}$ is the free Dirac Hamiltonian, the operator $V$ describes the electromagnetic interaction between the bound electron and the nucleus, and $H=H_{0}+V$ is the total Hamiltonian. The corresponding diagrams are presented in Fig. 3. The three terms in Eq. (12) give three corresponding contributions to the SE-WF determined by Eq. (4): the zeropotential, one-potential, and many-potential terms, which will be described in detail in the following subsections.

\section{A. Zero-potential contribution}

Let us start with the zero-potential term, i.e., the part where there is no interaction between the electron and the nucleus

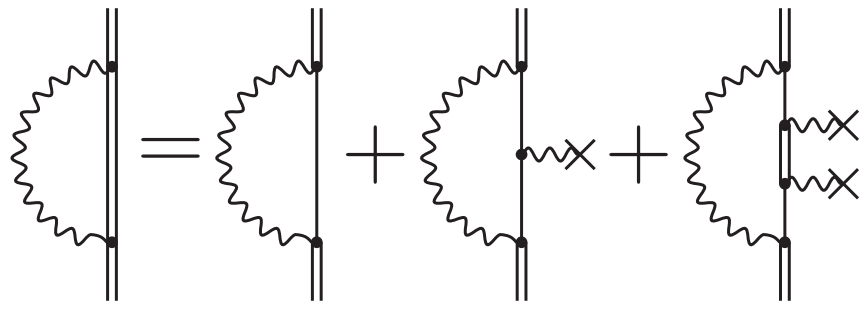

FIG. 3. Expansion of the electron propagator within the selfenergy diagram in powers of interactions with the nucleus. Single lines represent free-electron propagators; a wavy line terminated by a cross represents an interaction with the nucleus.

inside the SE loop, corresponding to the first term in Eq. (12) and in Fig. 3. Following the procedure described in Ref. [29], and taking into account the mass counterterm, one can write the following expression for the zero-potential contribution to the nondiagonal SE matrix element (see Fig. 3) in the momentum representation:

$$
\Delta E_{a b}^{(0)}=\int \frac{d \mathbf{p}}{(2 \pi)^{3}} \bar{\psi}_{a}(\mathbf{p})\left(\Sigma^{(0)}(\mathrm{p})-\delta m\right) \psi_{b}(\mathbf{p}) .
$$

Here $\bar{\psi}=\psi^{\dagger} \gamma^{0}$, and $\Sigma^{(0)}$ is the free SE function [29]. The electronic wave function in the momentum representation is determined via the Fourier transform

$$
\psi(\mathbf{p})=\int d \mathbf{r} e^{-i \mathbf{p} \cdot \mathbf{r}} \psi(\mathbf{r})=i^{-l}\left(\begin{array}{c}
g(p) \Omega_{\kappa m}(\widehat{\mathbf{p}}) \\
f(p) \Omega_{-\kappa m}(\widehat{\mathbf{p}})
\end{array}\right),
$$

with the radial wave functions $g(p)$ and $f(p)$, and the angular part given by spherical spinors $\Omega_{\kappa m}$, like in the coordinate representation (1). The normalization integral for the momentumrepresentation wave functions reads

$$
\frac{1}{(2 \pi)^{3}} \int d p p^{2}\left[g^{2}(p)+f^{2}(p)\right]=1 .
$$

Note that after subtraction of the mass counterterm the zero-potential part of the SE operator still contains a UVdivergent contribution:

$$
\Sigma^{(0)}(\mathrm{p})-\delta m=-\frac{\alpha}{4 \pi} \Delta_{\epsilon}(p-m)+\Sigma_{R}^{(0)}(\mathrm{p}),
$$

within the dimensional regularization procedure with the dimension $D=4-2 \epsilon$. Here, $p=\mathrm{p}_{\mu} \gamma^{\mu}, \Delta_{\epsilon}=\frac{1}{\epsilon}-\gamma_{E}+$ $\ln 4 \pi, \epsilon$ is a small parameter, and $\gamma_{E}$ is the Euler constant. When the SE operator acts on the bound-electron wave function, this divergent term is canceled by a matching UV divergence in the one-potential contribution, carried by the time-component $\Gamma^{0}$ of the free vertex function:

$$
\Gamma^{0}\left(\mathrm{p}^{\prime}, \mathrm{p}\right)=\frac{\alpha}{4 \pi} \Delta_{\epsilon} \gamma^{0}+\Gamma_{R}^{0}\left(\mathrm{p}^{\prime}, \mathrm{p}\right)
$$

which will be considered in the next subsection. For the calculation of the SE-WF, these remaining divergences in the zero- and one-potential contributions can be ignored due to this cancellation, and calculations can be performed for the renormalized (finite) remainders $\Sigma_{R}^{(0)}$ and $\Gamma_{R}^{(0)}$. 
Finally, the renormalized operator $\Sigma_{R}^{(0)}$ in the momentum representation reads

$\Sigma_{R}^{(0)}(\mathrm{p})=\frac{\alpha}{4 \pi}\left[2+\frac{4 \rho \ln \rho}{1-\rho}-\not p \frac{2-\rho}{1-\rho}\left(1+\frac{\rho \ln \rho}{1-\rho}\right)\right]$,

where $\rho=1-\mathrm{p}^{2}$. Taking into account the following identity obeyed by spherical spinors,

$$
(\sigma \cdot \widehat{\mathbf{p}}) \Omega_{\kappa \mu}(\widehat{\mathbf{p}})=-\Omega_{-\kappa \mu}(\widehat{\mathbf{p}}),
$$

we can write the final expression for the nondiagonal zeropotential term as follows:

$$
\begin{aligned}
\Delta E_{a b}^{(0)}= & \frac{\alpha}{4 \pi} \int_{0}^{\infty} \frac{d p p^{2}}{(2 \pi)^{3}}\left\{a(\rho)\left(g_{a} g_{b}-f_{a} f_{b}\right)\right. \\
& \left.+b(\rho)\left[\varepsilon_{a}\left(g_{a} g_{b}+f_{a} f_{b}\right)+p\left(g_{a} f_{b}+f_{a} g_{b}\right)\right]\right\}, \\
a(\rho)= & 2\left[1+\frac{2 \rho}{1-\rho} \ln \rho\right], \\
b(\rho)= & -\frac{2-\rho}{1-\rho}\left[1+\frac{\rho}{1-\rho} \ln \rho\right] .
\end{aligned}
$$

The radial wave functions are calculated at momentum $p$ : $g=g(p), f=f(p)$. Please note that the SE operator is diagonal in $\kappa$ and the angular momentum projection, and it does not change the energy, therefore only the reference-state energy (in our case state $a$ ) appears in the formula.

The calculation of the zero-potential contribution (17) is completed by the numerical evaluation of the one-dimensional integral in momentum space. The details of this calculation are described in Sec. III B.

\section{B. One-potential contribution}

The one-potential term has one single interaction between the electron and the nucleus inside the SE loop, corresponding to the second term in Eq. (12) and in Fig. 3. The renormalized expression for the one-potential term can be written in momentum representation as [25,29]

$$
\begin{aligned}
\Delta E_{a b}^{(1)} & =\int \frac{d \mathbf{p}^{\prime}}{(2 \pi)^{3}} \int \frac{d \mathbf{p}}{(2 \pi)^{3}} \\
& \times \bar{\psi}_{a}\left(\mathbf{p}^{\prime}\right) \Gamma_{R}^{0}\left(\mathrm{p}^{\prime}, \mathrm{p}\right) V\left(\mathbf{p}^{\prime}-\mathbf{p}\right) \psi_{b}(\mathbf{p}) .
\end{aligned}
$$

Here the renormalized vertex function is

$$
\begin{aligned}
\Gamma_{R}^{\mu}\left(\mathrm{p}^{\prime}, \mathrm{p}\right)= & \frac{\alpha}{4 \pi}\left[A \gamma^{\mu}+\not p^{\prime}\left(B_{1} \mathrm{p}^{\prime \mu}+B_{2} \mathrm{p}^{\mu}\right)\right. \\
& \left.+\not C_{1} \mathrm{p}^{\prime \mu}+C_{2} \mathrm{p}^{\mu}\right)+D \not^{\prime} \gamma^{\mu} \not \\
& \left.+\left(H_{1} \mathrm{p}^{\prime \mu}+H_{2} \mathrm{p}^{\mu}\right)\right] .
\end{aligned}
$$

The coefficients are given as

$$
\begin{aligned}
A= & C_{24}-2+\mathrm{p}^{\prime 2} C_{11}+\mathrm{p}^{2} C_{12} \\
& +4\left(\mathrm{p}^{\prime} \cdot \mathrm{p}\right)\left(C_{0}+C_{11}+C_{12}\right) \\
& -2 C_{0}+C_{11}+C_{12}, \\
B_{1}= & -4\left(C_{11}+C_{21}\right), \\
B_{2}= & -4\left(C_{0}+C_{11}+C_{12}+C_{23}\right), \\
C_{1}= & -4\left(C_{0}+C_{11}+C_{12}+C_{23}\right), \\
C_{2}= & -4\left(C_{12}+C_{22}\right),
\end{aligned}
$$

$$
\begin{aligned}
& D=2\left(C_{0}+C_{11}+C_{12}\right), \\
& H_{1}=4\left(C_{0}+2 C_{11}\right), \\
& H_{2}=4\left(C_{0}+2 C_{12}\right),
\end{aligned}
$$

via the one-parameter Feynman integrals

$$
\begin{aligned}
C_{0}= & \int_{0}^{1} \frac{d y}{\left(y \mathrm{p}^{\prime}+(1-y) \mathrm{p}\right)^{2}}(-\ln X) \\
\left(\begin{array}{l}
C_{11} \\
C_{12}
\end{array}\right)= & \int_{0}^{1} \frac{d y}{\left(y \mathrm{p}^{\prime}+(1-y) \mathrm{p}\right)^{2}}\left(\begin{array}{c}
y \\
1-y
\end{array}\right) \\
& \times(1-Y \ln X) \\
\left(\begin{array}{l}
C_{21} \\
C_{22} \\
C_{23}
\end{array}\right)= & \int_{0}^{1} \frac{d y}{\left(y \mathrm{p}^{\prime}+(1-y) \mathrm{p}\right)^{2}}\left(\begin{array}{c}
y^{2} \\
(1-y)^{2} \\
y(1-y)
\end{array}\right) \\
& \times\left(-\frac{1}{2}+Y-Y^{2} \ln X\right) \\
C_{24}= & -\int_{0}^{1} d y \ln \left(y^{2}\left(\mathrm{p}^{\prime}-\mathrm{p}\right)^{2}-y\left(\mathrm{p}^{\prime}-\mathrm{p}\right)^{2}+1\right)
\end{aligned}
$$

We used the notations

$$
\begin{aligned}
X & =1+\frac{1}{Y}, \\
Y & =\frac{1-y \mathrm{p}^{\prime 2}-(1-y) \mathrm{p}^{2}}{\left[y \mathrm{p}^{\prime}+(1-y) \mathrm{p}\right]^{2}} .
\end{aligned}
$$

Note that the denominators in Eqs. (22) can be equal to zero. However, this does not lead to a singularity in the expressions of Eqs. (21), since the zeros of the denominator are also zeros of the numerator of the same power.

The final renormalized expression for the nondiagonal onepotential contribution is

$$
\begin{aligned}
\Delta E_{a b}^{(1)}= & \frac{\alpha}{2(2 \pi)^{6}} \int_{0}^{\infty} d p p^{2} \int_{0}^{\infty} d p^{\prime} p^{2} \\
& \times \int_{-1}^{1} d \xi V(q)\left[\mathcal{F}_{1}^{a b} P_{l_{a}}(\xi)+\mathcal{F}_{2}^{a b} P_{l_{a}}(\xi)\right],
\end{aligned}
$$

where $q^{2}=p^{2}+p^{\prime 2}-2 p p^{\prime} \xi, \bar{l}=2 j-l, P_{l}$ are the Legendre polynomials, and

$$
\begin{aligned}
\mathcal{F}_{1}^{a b}= & A g_{a}^{\prime} g_{b}+\left(B_{1} \varepsilon_{a}+B_{2} \varepsilon_{b}\right)\left(\varepsilon_{a} g_{a}^{\prime}+p^{\prime} f_{a}^{\prime}\right) g_{b} \\
& +\left(C_{1} \varepsilon_{a}+C_{2} \varepsilon_{b}\right) g_{a}^{\prime}\left(\varepsilon_{b} g_{b}+p f_{b}\right) \\
& +D\left(\varepsilon_{a} g_{a}^{\prime}+p^{\prime} f_{a}^{\prime}\right)\left(\varepsilon_{b} g_{b}+p f_{b}\right)+\left(H_{1} \varepsilon_{a}+H_{2} \varepsilon_{b}\right) g_{a}^{\prime} g_{b},
\end{aligned}
$$

$$
\begin{aligned}
\mathcal{F}_{2}^{a b}= & A f_{a}^{\prime} f_{b}+\left(B_{1} \varepsilon_{a}+B_{2} \varepsilon_{b}\right)\left(\varepsilon_{a} f_{a}^{\prime}+p^{\prime} g_{a}^{\prime}\right) f_{b} \\
& +\left(C_{1} \varepsilon_{a}+C_{2} \varepsilon_{b}\right) f_{a}^{\prime}\left(\varepsilon_{b} f_{b}+p g_{b}\right) \\
& +D\left(\varepsilon_{a} f_{a}^{\prime}+p^{\prime} g_{a}^{\prime}\right)\left(\varepsilon_{b} f_{b}+p g_{b}\right)-\left(H_{1} \varepsilon_{a}+H_{2} \varepsilon_{b}\right) f_{a}^{\prime} f_{b} .
\end{aligned}
$$

The momentum-representation radial wave functions have to be calculated at points $p$ and $p^{\prime}$ for unprimed and primed variables, correspondingly: $g=g(p), g^{\prime}=g\left(p^{\prime}\right)$.

For the numerical evaluation of the one-potential term, one has to calculate a four-dimensional integral, with an integrable 
singularity at the point $\xi=1$, and the artificial singularities originating from the zeros in the denominator. Around these points, a Taylor expansion is used, which allows us to work with an explicitly regular expression. Further details will be given in Sec. III C.

\section{Many-potential contribution}

Finally, let us consider the remaining many-potential part with two or more interactions between the electron and the nucleus inside the SE loop, corresponding to the third term in Eq. (12) and in Fig. 3. The many-potential contribution is calculated in the coordinate representation by the formula

$$
\begin{aligned}
\Delta E_{a b}^{(2+)}= & \frac{i}{2 \pi} \int_{-\infty}^{\infty} d \omega\left[\sum_{n} \frac{\langle a n|I(\omega)| n b\rangle}{\varepsilon_{a}-\omega-\varepsilon_{n}(1-i 0)}\right. \\
& - \text { subtractions }] .
\end{aligned}
$$

The first term corresponds to the nonrenormalized contribution [see Eq. (2)], whereas the subtractions account for $G^{(0)}$ and $G^{(1)}$, namely, correspond to the substitution of the bound Green's function $G \rightarrow G-G^{(0)}-G^{(1)}$. Here, $G^{(0)}$ is a free-electron propagator, and $G^{(1)}$ contains one interaction with the field $V$, and in the case of the Coulomb field can be calculated as the derivative $G^{(1)}=\left.Z G^{\prime}(Z)\right|_{Z=0}$. In our calculations, however, the following alternative expression for the many-potential term was used, as it was proposed in Ref. [41]:

$$
\begin{gathered}
\Delta E_{a b}^{(2+)}=\frac{i}{2 \pi} \int_{-\infty}^{\infty} d \omega \sum_{n} \frac{\left\langle a n^{\prime}|I(\omega)| n^{\prime} b\right\rangle}{\varepsilon_{a}-\omega-\varepsilon_{n}(1-i 0)}, \\
\left|n^{\prime}\right\rangle=\sum_{f} \frac{|f\rangle\langle f|V| n\rangle}{\varepsilon_{a}-\omega-\varepsilon_{f}(1-i 0)} .
\end{gathered}
$$

Here $|n\rangle$ is a bound state in the external potential $V$, and $|f\rangle$ belongs to the spectrum of the free electron. Angular integrations can be performed via the partial wave expansion of the interelectronic interaction operator $I(\omega)$ as follows (see, e.g., [29]):

$$
\langle a b|I(\omega)| c d\rangle=\alpha \sum_{J=0}^{\infty} I_{J}(a b c d) R_{J}(\omega, a b c d) .
$$

$I_{J}$ contains the full dependence on the angular momentum projections via $3 j$ symbols [42]

$$
\begin{aligned}
I_{J}(a b c d)= & \sum_{M}(-1)^{j_{a}-m_{a}+J-M+j_{b}-m_{b}} \\
& \times\left(\begin{array}{ccc}
j_{a} & J & j_{c} \\
-m_{a} & M & m_{c}
\end{array}\right)\left(\begin{array}{ccc}
j_{b} & J & j_{d} \\
-m_{b} & -M & m_{d}
\end{array}\right),
\end{aligned}
$$

and $R_{J}$ are generalized Slater radial integrals:

$$
\begin{aligned}
R_{J}(\omega, a b c d) & \\
= & (2 J+1) \int_{0}^{\infty} d r_{1} d r_{2} r_{1}^{2} r_{2}^{2} \\
& \times\left[(-1)^{J} g_{J}\left(\omega, r_{<}, r_{>}\right) W_{a c, J}\left(r_{1}\right) W_{b d, J}\left(r_{2}\right)\right. \\
& \left.-\sum_{L}(-1)^{L} g_{L}\left(\omega, r_{<}, r_{>}\right) X_{a c, J L}\left(r_{1}\right) X_{b d, J L}\left(r_{2}\right)\right],
\end{aligned}
$$

where $\quad r_{<}=\min \left(r_{1}, r_{2}\right), \quad r_{>}=\max \left(r_{1}, r_{2}\right), \quad$ and $\quad g_{L}(\omega$, $\left.r_{<}, r_{>}\right)=i \omega j_{L}\left(\omega r_{<}\right) h_{L}^{(1)}\left(\omega r_{>}\right)$with the spherical Bessel functions $j_{L}$ and $h_{L}^{(1)}$. We have used the following notations in the radial integrals,

$$
\begin{aligned}
W_{a b, J}(r)= & C_{J}\left(\kappa_{a}, \kappa_{b}\right)\left[g_{a}(r) g_{b}(r)+f_{a}(r) f_{b}(r)\right], \\
X_{a b, J L}(r)= & g_{a}(r) f_{b}(r) S_{J L}\left(-\kappa_{b}, \kappa_{a}\right) \\
& -f_{a}(r) g_{b}(r) S_{J L}\left(\kappa_{b},-\kappa_{a}\right),
\end{aligned}
$$

with the nonvanishing angular coefficients

$$
\begin{aligned}
C_{J}\left(\kappa_{1}, \kappa_{2}\right)= & \sqrt{\left(2 l_{1}+1\right)\left(2 l_{2}+1\right)\left(2 j_{1}+1\right)\left(2 j_{2}+1\right)} \\
& \times(-1)^{j_{2}+J+1 / 2}\left(\begin{array}{ccc}
l_{1} & J & l_{2} \\
0 & 0 & 0
\end{array}\right)\left\{\begin{array}{lll}
j_{1} & j_{2} & J \\
l_{2} & l_{1} & 1 / 2
\end{array}\right\}, \\
S_{J J+1}\left(\kappa_{1}, \kappa_{2}\right)= & \sqrt{\frac{J+1}{2 J+1}}\left(1+\frac{\kappa_{1}+\kappa_{2}}{J+1}\right) C_{J}\left(-\kappa_{2}, \kappa_{1}\right), \\
S_{J J}\left(\kappa_{1}, \kappa_{2}\right)= & \frac{\kappa_{1}-\kappa_{2}}{\sqrt{J(J+1)}} C_{J}\left(\kappa_{2}, \kappa_{1}\right), \\
S_{J J-1}\left(\kappa_{1}, \kappa_{2}\right)= & \sqrt{\frac{J}{2 J+1}}\left(-1+\frac{\kappa_{1}+\kappa_{2}}{J}\right) C_{J}\left(-\kappa_{2}, \kappa_{1}\right) .
\end{aligned}
$$

Performing analytically the angular integrations and summations over the projections in Eq. (26), we obtain a significantly simplified expression for the many-potential term:

$$
\begin{aligned}
\Delta E_{a b}^{(2+)}= & \frac{i \alpha}{2 \pi} \int_{-\infty}^{\infty} d \omega \sum_{n J} \frac{(-1)^{j_{n}-j_{a}+J}}{2 j_{a}+1} \\
& \times \frac{R_{J}\left(\omega, a n^{\prime} n^{\prime} b\right)}{\varepsilon_{a}-\omega-\varepsilon_{n}(1-i 0)} .
\end{aligned}
$$

The sum over $n$ in this expression corresponds to the summation over the principal and relativistic angular quantum numbers of the intermediate states. The details of the numerical calculation will be discussed in Sec. III D.

\section{NUMERICAL CALCULATIONS}

To solve the Dirac equation numerically we used the dual-kinetic balance (DKB) method [43] based on B-splines [44,45]. This method provides solutions for an arbitrary spherically symmetric potential. The solutions are determined in a cavity, which allows one to have a finite number of electronic states, representing the full spectrum, including negative and positive continuum states, for a given value of the relativistic angular momentum number $\kappa$. Low-lying bound states can be reproduced with high accuracy. Thanks to the availability of this discrete numerical spectrum, it is possible to calculate sums over the spectrum such as those featured in Eq. (4). In our calculations, we used a homogeneously charged sphere model for the charge distribution inside of the nucleus. The root-mean-square nuclear radii were taken from Ref. [46].

\section{A. Fourier transform}

The calculation of the zero- and one-potential terms has to be performed in momentum space [see Eqs. (17) and (23), 
respectively]. Therefore, the calculation of the SE-WF requires electron wave functions in the momentum representation. As it turns out, this is a major difficulty. Since the momentum-representation wave functions are determined via the Fourier transform (14), the radial wave functions in momentum space can be calculated with a generalized Fourier transform:

$$
\begin{aligned}
& g(p)=\sqrt{\frac{2}{\pi}} \int_{0}^{\infty} d r r^{2} j_{l}(p r) g(r), \\
& f(p)=-\frac{\kappa}{|\kappa|} \sqrt{\frac{2}{\pi}} \int_{0}^{\infty} d r r^{2} j_{\bar{l}}(p r) f(r) .
\end{aligned}
$$

The spherical Bessel functions $j_{l}(x)$ oscillate very fast for large arguments of $x$, making the numerical integration extremely slow and poorly converging. However, the wave functions in the DKB approach are represented by piecewise polynomials on intervals $\left[r_{i}, r_{i+1}\right]$, which allows us to use a semianalytical approach in the calculation of the oscillating radial integrals Eqs. (34). The integral of the Bessel function with a monomial function on a given interval can be calculated analytically:

$$
\int_{a}^{b} j_{n}(r) r^{m} d x=\text { Function }(a, b, n, m) .
$$

Therefore, on every interval $\left[r_{i}, r_{i+1}\right]$, the integrals (34) can be calculated as a sum of the analytically calculated integrals for the different powers of $r$. However, for large values of $r$, the coefficients of such an expansion in monomials can be very large, resulting in very large cancellations between different contributions, which leads to large numerical errors. For this reason, we do not separate the wave functions into their polynomial pieces. Rather, we use the full expression for the wave functions on each of the intervals for the integration and then add the results of each of the intervals.

As already noted, the generalized Fourier transform is a highly oscillatory integral for large enough momenta. To handle this complication, we express the spherical Bessel functions in terms of sine and cosine functions. That is, we express the generalized Fourier transforms as sums of sine and cosine transforms on finite intervals of appropriate functions. We then use routines from the numerical integration library QUADPACK [47] dedicated for the computation of these kinds of sine and cosine transforms. These routines allow us to set target accuracy values to control the accuracy of the result.

In our calculations, we requested the relative and the absolute accuracy of the calculations to be $10^{-6}$ and $10^{-15}$, respectively. However, this accuracy could not always be reached due to the complexity of the calculations, especially for large values of integration variables. Therefore in addition to the accuracy control in our calculations, we also carried out a few tests to estimate the resulting SE-WF (see Sec. V for details).

\section{B. Zero-potential contribution}

The calculation of the zero-potential contribution (17) to the SE-WF (4) can be performed in two steps. First, one calculates the corresponding nondiagonal contributions $\Delta E_{n a}^{(0)}$ for the full spectrum of the electronic states $n$. Thereafter, the zero-potential contribution to the SE-WF can be written as a point-by-point summation of those contributions divided by the energy difference:

$$
\left|\delta_{\mathrm{SE}}^{(0)} a\right\rangle=\sum_{n}^{\prime} \frac{|n\rangle \Delta E_{n a}^{(0)}}{\varepsilon_{a}-\varepsilon_{n}} .
$$

However, this straightforward approach was found to be inapplicable here. In addition to the oscillations in the spherical Bessel functions, the wave functions corresponding to the positive and negative continua in the discrete numerical spectra of the B-splines also contain significant oscillations towards the end of the cavity. Combined together, these factors make the direct calculation of the integrals $\Delta E_{n a}^{(0)}$ impossible with any reasonable accuracy. To overcome this obstacle, an alternative approach based on the reduced Green's function has been used. The radial part of the reduced Green's function as a function of the reference state energy and two coordinates can be calculated as

$$
G^{\prime}\left(\varepsilon_{a}, \mathbf{r}_{1}, \mathbf{r}_{2}\right)=\sum_{n}^{\prime} \frac{|n\rangle\langle n|}{\varepsilon_{a}-\varepsilon_{n}} .
$$

Then, the zero-potential contribution to the SE-WF can be described by the expression

$$
\left|\delta_{\mathrm{SE}}^{(0)} a\right\rangle=G^{\prime}\left(\varepsilon_{a}\right) \Sigma_{R}^{(0)}|a\rangle .
$$

The reduced Green's function $G^{\prime}\left(\varepsilon_{a}, \mathbf{r}_{1}, \mathbf{r}_{2}\right)$ has a smooth dependence on radial variables which greatly simplifies the numerical Bessel transform (34). Since all the wave functions have piecewise polynomial structure, the Green's function, as a linear combination of the wave functions, also has the same structure. Therefore, the procedure described in the previous subsection can be applied here.

Finally, the full procedure functions as follows. For each point of the predefined grid on $r_{1}$ we calculate the radial part of the reduced Green's function $G^{\prime}\left(\varepsilon_{a}, r_{1}, r_{2}\right)$. Then we perform the Fourier transform over the second variable as

$$
G^{\prime}\left(\varepsilon_{a}, r_{1}, r_{2}\right) \rightarrow G^{\prime}\left(\varepsilon_{a}, r_{1}, p\right) .
$$

Finally, to obtain the zero-potential part of our SE-WF in coordinate representation as a function of $r_{1}$, we integrate over $p$ in Eq. (37), using the zero-potential matrix element (17) of the SE operator. In the case of the diagonal matrix element $\Delta E_{a a}^{(0)}$, a comparably sparse integration grid on $p$ can be used. For the SE-WF calculation, due to discontinuity of the radial Green's function $G\left(\varepsilon_{a}, r_{1}, r_{2}\right)$ at $r_{1}=r_{2}$, we had to break the radial integration over $r_{2}$ at this point and, therefore, different values of $r_{1}$ required different radial grids for $r_{2}$. Therefore, the integration has been performed with a DQAGI procedure from the QUADPACK package with target accuracy values to control the accuracy of the result.

\section{One-potential contribution}

The calculation of the one-potential contribution to the SE-WF is in principle similar to that of the zero-potential term. Again, instead of calculating the set of nondiagonal contributions $\Delta E_{n a}^{(1)}$ and then summing point by point over 
the whole spectrum according to

$$
\left|\delta_{\mathrm{SE}}^{(1)} a\right\rangle=\sum_{n}^{\prime} \frac{|n\rangle \Delta E_{n a}^{(1)}}{\varepsilon_{a}-\varepsilon_{n}},
$$

we first calculate the reduced Green's function and then evaluate the one-potential contribution with its help according to

$$
\left|\delta_{\mathrm{SE}}^{(1)} a\right\rangle=G^{\prime}\left(\varepsilon_{a}\right) \Gamma_{R}^{(0)} V|a\rangle .
$$

Therefore, the numerical integration in Eq. (23) has to be performed for a given point of the reduced Green's function $G^{\prime}\left(\varepsilon_{a}, r_{1}, p\right)$, and the integrals calculated independently for the lower and upper components of the resulting SE-WF. In comparison with the zero-potential term, the difference is that instead of a regular one-dimensional integral we now have a four-dimensional integrand featuring integrable singularities. The first singularities are the zeros of the denominators of the Feynman integrals in Eqs. (17). To integrate around these points, a Taylor expansion was used, giving rise to analytically simplified expressions, which do not contain any singularities anymore. The next problem arises at point $q=0$, which occurs when $\xi=1$ and $p_{1}=p_{2}$. The singularity comes from the momentum representation of the nuclear potential, determined as an integral:

$$
\begin{aligned}
V(p) & =\int d \mathbf{r} \exp (-i \mathbf{p} \cdot \mathbf{r}) V(\mathrm{r}) \\
& =4 \pi \int_{0}^{\infty} d r r^{2} j_{0}(p r) V(r) .
\end{aligned}
$$

For the pointlike nucleus, the Fourier transform of the Coulomb potential $V_{\text {point }}(r)=-\alpha Z / r$ can be used:

$$
V_{\text {point }}(p)=-\frac{4 \pi \alpha Z}{p^{2}} .
$$

In the case of the homogeneously charged sphere nuclear model with the radius $r_{N}$, the expression is less trivial:

$$
V_{\mathrm{sph}}(p)=-\frac{3 \alpha Z}{p^{2}} \frac{\sin \left(p r_{N}\right)-p r_{N} \cos \left(p r_{N}\right)}{\left(p r_{N}\right)^{3}}
$$

In the case of other more complicated potentials, i.e., Fermi or any effective potentials, including electron screening or a Uehling potential, there are no analytical formulas, and the momentum representation of the nuclear potential has to be calculated numerically using Eq. (40).

For the numerical integration in Eq. (17), it is more convenient to change the variable from $\xi \in[-1,1]$ to $q=p_{1}^{2}+$ $p_{2}^{2}+2 \xi p_{1} p_{2}, q \in\left[\left|p_{1}-p_{2}\right|, p_{1}+p_{2}\right]$, as it was suggested in Ref. [29]. Then the integral contains an integrable singularity of $1 / q$. The integrations over the Feynman parameters and over $q$ were performed with the DQAGS procedure. The remaining two integrations over $p_{1}$ and $p_{2}$ on the infinite intervals $[0, \infty)$ were performed again with DQAGI.

\section{Many-potential contribution}

For the calculation of the many-potential contribution to the SE-WF a straightforward approach has been used: we start

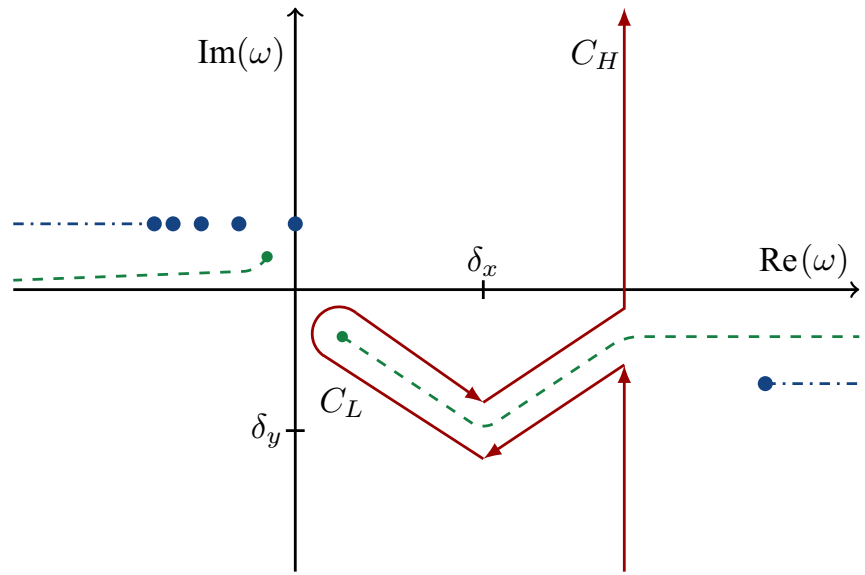

FIG. 4. The singularities of the integrand (blue and green dots and lines) of the many-potential part Eq. (43) and the modified (in the most general form) integration contour (red line) for the $1 s$ state; see the text and Ref. [17] for further detail.

from

$$
\left|\delta_{\mathrm{SE}}^{(2+)} a\right\rangle=\sum_{n}^{\prime} \frac{|n\rangle \Delta E_{n a}^{(2+)}}{\varepsilon_{a}-\varepsilon_{n}} .
$$

For each state $n$ in the spectrum the nondiagonal element defined by Eq. (26) can be calculated independently, and the SE-WF calculated as a point-by-point summation after this.

The following numerical difficulties arise for the manypotential contribution: First of all, the integrand in Eq. (33) is again a highly oscillating function of $\omega$, which makes direct usage of that expression problematic. Additionally, in the complex plane, the integrand has poles at points $\omega=\varepsilon_{a}-$ $\varepsilon_{n}+i 0$, where $\left|\varepsilon_{n}\right|<m c^{2}$, and two branches starting from the points $\omega=\varepsilon_{a} \pm m c^{2}(1-i 0)$, originating from the bound electron's Green's functions, and two branch cuts starting from the points $\omega= \pm \sqrt{-i \delta}$, originating from the photon propagator. A schematic picture of the analytical structure of the integrand is given in Fig. 4 for the case of the reference state $1 s$. The simplest way to suppress the oscillations is the rotation of the integration contour as $\omega \rightarrow \pm i \omega$, so that the oscillating exponential function $\exp (i \omega x)$ turns into the decaying $\exp (-\omega x)$. However, this rotation would lead to the appearance of pole terms for all intermediate bound states with energies equal to or below the energy $\varepsilon_{a}$ of the considered state $a$. The integral contribution and the pole terms partly cancel each other. Therefore, this approach is reasonable to use for the ground or the first few excited states, where the number of poles and cancellations are not very large yet.

For a more general approach and in order to avoid the cancellations with the pole terms, one can carry out the integration over the more complicated contour in the complex plane shown in Fig. 4.

The contour consists of a low-energy part $C_{L}$ and a highenergy part $C_{H}$, as explained in Ref. [17]. In this case, the integration over the upper and lower branches of the cut leads to the following replacement in the numerical calculations of the low-energy part:

$$
g_{L}(\omega) \rightarrow g_{L}(\omega)-g_{L}(-\omega) .
$$




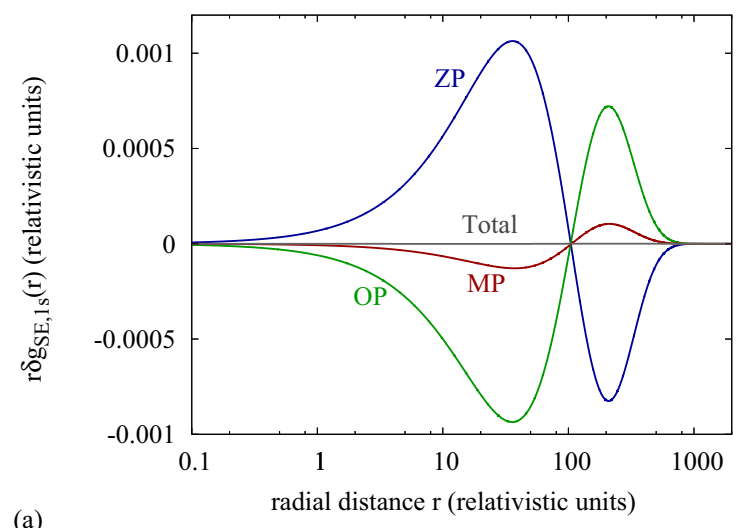

(a)

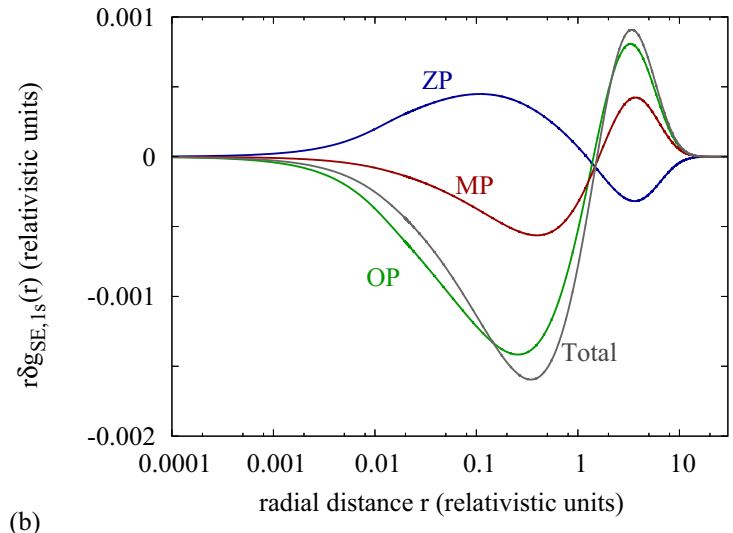

(b)

FIG. 5. Zero-potential (ZP, blue), one-potential (OP, green), and many-potential (MP, red) contributions, and the total value (gray) for the large component of the self-energy-corrected wave function $r \delta g_{\mathrm{SE}}(r)$ for the ground state of hydrogenlike ions with (a) $Z=2$ and (b) $Z=92$.

For the high energy-part, both contributions are equivalent, therefore a simple multiplication factor 2 is applied.

The summation over $n$ in Eq. (33) goes over the principal quantum number and also involves an infinite summation over the relativistic angular quantum number $\kappa$. In our numerical calculations, the summation is terminated at the maximum value $|\kappa|=18$, while the residual part of the sum is evaluated by a least-square inverse-polynomial fitting. For any given $\kappa$, the summation over the Dirac spectrum is performed utilizing the DKB approach [43] involving basis functions constructed from B-splines. For each value of $\omega$, the integrand still contains the summation over the spectrum $n^{\prime}$, the summation inside of it over the free-electron spectra $f$, and the summation over the partial waves $J$. Therefore, to increase the speed of the calculation, all wave functions, angular coefficients, and spherical Bessel functions have to be precalculated on the predetermined integration grid.

\section{RESULTS AND DISCUSSION}

In Fig. 5 we show the results of our calculation for different nuclear charge numbers $Z$ for the zero-, one-, and manypotential terms, plotting $r \delta g_{\mathrm{SE}}(r)$, the large component multiplied by the radius. As can be seen, the different contributions have different signs and partly cancel each other. As is clear from Fig. 5(b), the total SE-WF for $Z=92$ is of the same order of magnitude as the individual contributions. In contrast, for $Z=2$ there is a very large cancellation between the individual terms, and the total value is indistinguishable from zero in Fig. 5(a). Therefore, for $Z=2$ the absolute values of the individual contributions and the total value of the SE-WF are plotted in Fig. 6 on a logarithmic scale, revealing the cancellation of individual contributions up to four orders of magnitude.

For large radii, one can see oscillations in the SE-WF, which appear due to several factors. First of all, the boundelectron wave functions themselves have oscillations close to the edges of the box, even though the analytical expressions for the wave functions do not feature any. However, the magnitude of those oscillations is very small so that normally they do not limit the accuracy of calculations. Second, in the case of the zero- and one-potential terms, we have an additional numerical noise for large radial distances because of the fast oscillations in the spherical Bessel transform. For large $Z$ such as $Z=92$, the noise in the SE-WF is at least four orders of magnitude smaller than its value in the nuclear region, and it does not influence the calculations. For smaller $Z$, this is not the case. In the case of $Z=2$, the cancellations between the zero-, one-, and many-potential terms concern the first four digits. Therefore, even when these three contributions separately are calculated accurately, the total results feature significant numerical noise.

In order to solve this problem, we analyze the asymptotic behavior of the SE-WF for large radii $r$. By analyzing the SEWFs we found that they are proportional to the wave functions of the unperturbed state multiplied by $r$ :

$$
\left(\begin{array}{l}
\delta_{\mathrm{SE}} g_{n_{a} \kappa_{a}}(r) \\
\delta_{\mathrm{SE}} f_{n_{a} \kappa_{a}}(r)
\end{array}\right) \sim \frac{\alpha}{\pi}\left(\begin{array}{l}
r g_{n_{a} \kappa_{a}}(r) \\
r f_{n_{a} \kappa_{a}}(r)
\end{array}\right) .
$$

Therefore, the SE-WF obtained through our methods, even for small values of $Z$, still can be used. Fitting the SE-WF with the unperturbed wave functions multiplied by $r$ in a region where the SE-WF displays a smooth behavior, we were able to find a proportionality coefficient, and replace the SE-WF with

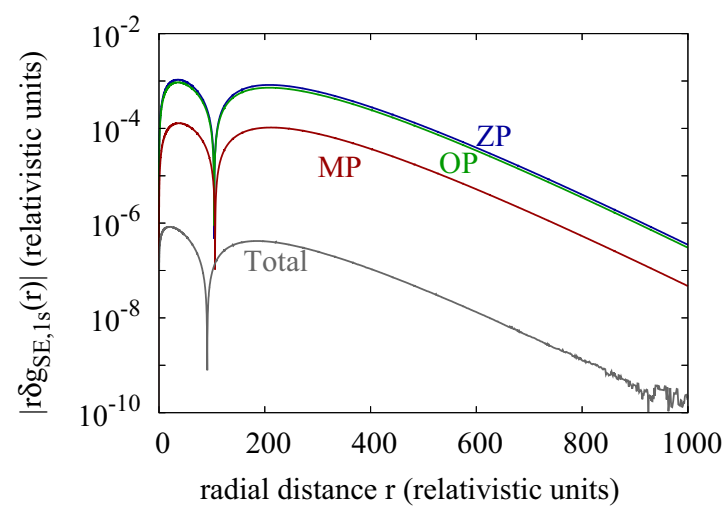

FIG. 6. Absolute values of the zero-potential (blue), onepotential (green), and many-potential (red) contributions, and of the total value (gray) for the large component of the self-energycorrected wave function $\left|r \delta g_{\mathrm{SE}}(r)\right|$ for the ground state of hydrogenlike ions with $Z=2$ in logarithmic scale. 

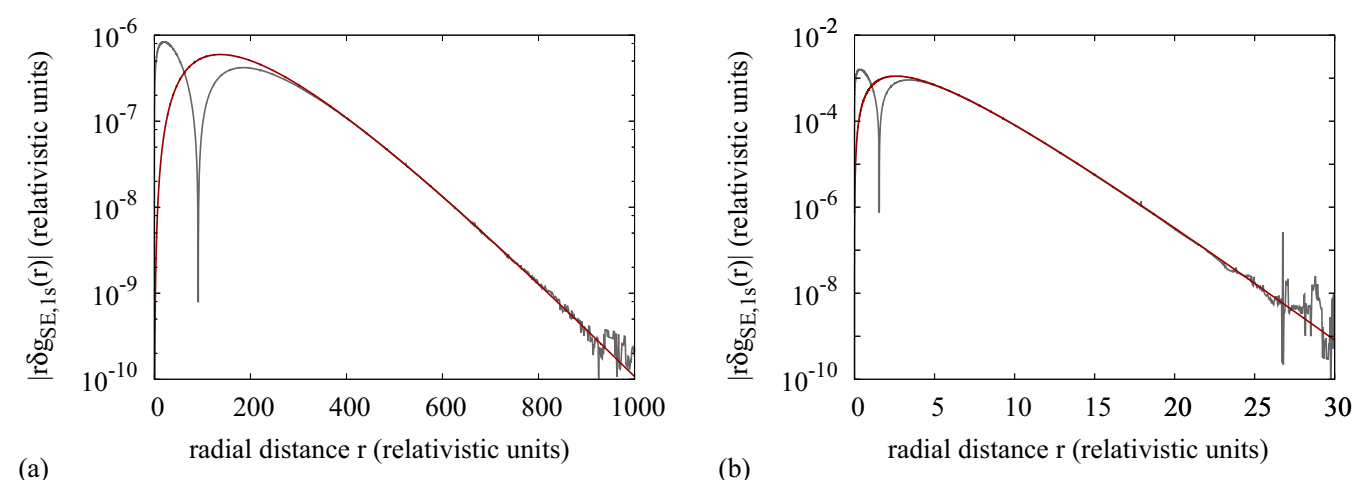

FIG. 7. Absolute values of the large component of the total self-energy-corrected wave function $r \delta g_{\mathrm{SE}}(r)$ (gray) and its extrapolation (red) according to Eq. (44) for the ground state of hydrogenlike ions with (a) $Z=2$ and (b) $Z=92$.

the corresponding asymptotic equivalent in the region where the numerical noise becomes too large. The total SE-WFs and their asymptotic extrapolation are shown in Fig. 7.

With these SE-WFs, we can also calculate the SE-WFs in the momentum representation, following the procedure described earlier in Sec. III A. The resulting SE-WFs are plotted in Fig. 8.

\section{CONSISTENCY TESTS}

\section{A. One-loop $g$ factor}

To check the accuracy of our SE-WFs, we used them to compute the irreducible part of the SE correction to the boundelectron $g$ factor [see Fig. 1(a)]. This correction is usually computed in the literature following Eq. (8), as the matrix element of the SE operator between the bound electron's wave function and the wave function perturbed by a constant homogeneous magnetic field B. Rewriting Eq. (9) for the $g$ factor, we computed the correction using the SE-WF instead, according to

$$
g_{\mathrm{SE}, \mathrm{wf}, \text { irred }}=-\frac{8}{e B}\left\langle a|(-e \boldsymbol{\alpha} \cdot \mathbf{A})| \delta_{\mathrm{SE}} a\right\rangle .
$$

Here, we assume the angular momentum projection to be $1 / 2$, and the prefactor $-8 / e B$ comes from converting from the energy shift to the $g$ factor.

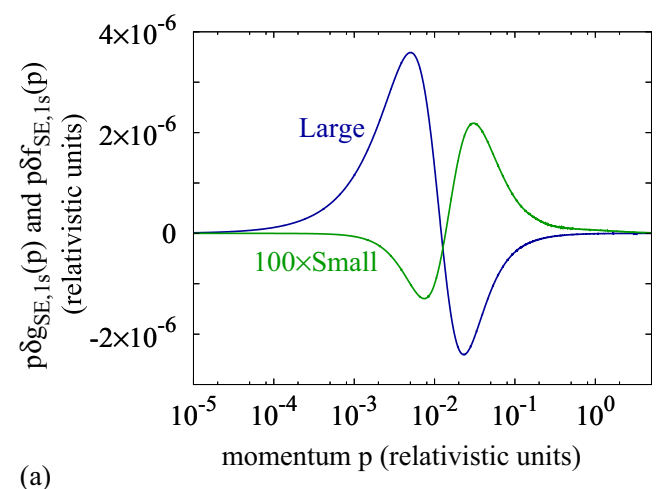

(a)

FIG. 8. The large (blue) $p \delta g_{\mathrm{SE}}(p)$ and the small (green) $p \delta f_{\mathrm{SE}}(p)$ components of the total self-energy-corrected wave function in momentum representation for the ground state of hydrogenlike ions with (a) $Z=2$ and (b) $Z=92$. For $Z=2$, the small component has been multiplied by 100 on the graph.
In Table I, we compare our results, obtained with Eq. (45), with the values given in Ref. [15]. We used Ref. [15] for comparison because in that work, just as in our case, calculations were performed for extended nuclei. Typically, we found a three- to four-digit agreement both with the coordinate and the momentum space representations of our SE-WFs.

\section{B. SE loop-after-loop correction}

We also computed the SE LAL contribution to the Lamb shift [first diagram in Fig. 1(b)]. Using the SE-WF, the energy shift according to Eq. (11) reads

$$
\Delta E_{\mathrm{LAL}}=\left\langle a\left|\Sigma_{R}\right| \delta_{\mathrm{SE}} a\right\rangle .
$$

The SE operator in Eq. (46) needs to be split again into the zero-, one-, and many-potential parts. The evaluation of the zero- and one-potential contributions to $\Delta E_{\mathrm{LAL}}$ requires the momentum representations of the bound-electron wave function and the SE-WF. The many-potential contribution to $\Delta E_{\mathrm{LAL}}$ can be computed in coordinate space. Table II shows a comparison of our calculation of $\Delta E_{\mathrm{LAL}}$ with values taken from Refs. [16,17].

We observe that for low and middle $Z$ our result agrees well with the values in Refs. [16,17], while there is a slight disagreement for $Z=92$. This discrepancy is due to the fact that, in our case, the SE-WF was obtained for extended nuclei, while the calculations in Ref. [17] are based on the pointlike

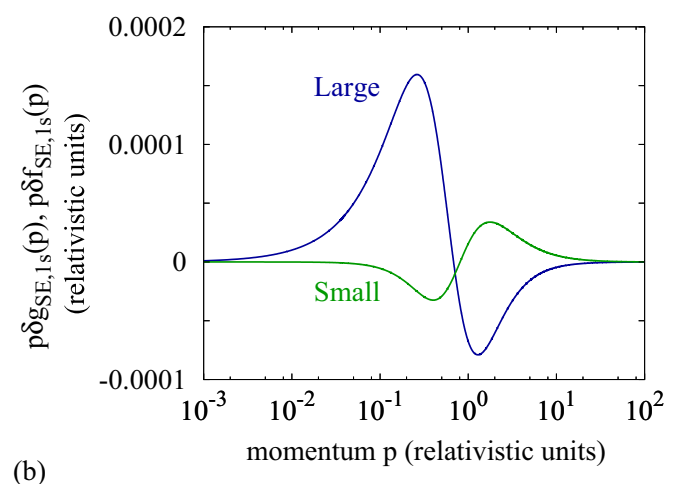

(b) 
TABLE I. $g_{\mathrm{SE}, \mathrm{wf} \text {,irred }}$ contribution to the ground-state $1 s$ boundelectron $g$ factor in units of $10^{-6}$. The first and second column contain our results according to Eq. (45), obtained with the coordinate and momentum representations of the SE-WF, respectively. The third column contains the values from Ref. [15].

\begin{tabular}{lccr}
\hline \hline$Z$ & Coord. repr. & Momen. repr. & \multicolumn{1}{c}{ Ref. [15] } \\
\hline 2 & $5.211(19)$ & $5.198(11)$ & $5.2065(4)$ \\
10 & $78.748(8)$ & $78.746(5)$ & $78.7434(4)$ \\
18 & $199.765(8)$ & $199.764(8)$ & $199.7635(4)$ \\
36 & $576.522(11)$ & $576.522(6)$ & $576.469(2)$ \\
54 & $1074.31(7)$ & $1074.30(7)$ & $1074.185(2)$ \\
92 & $2694.62(5)$ & $2694.63(5)$ & $2694.56(1)$ \\
\hline
\end{tabular}

nuclear model. With a smaller radius in our calculations, our results converge to those presented in Ref. [17].

\section{CONCLUSIONS AND OUTLOOK}

In this paper, we calculated the self-energy-corrected ground-state wave functions for a number of hydrogenlike ions. The general formulas, the numerical procedure, the problems encountered, and the methods employed to solve them are discussed in detail in the paper. Our tests show good agreement with previously published results and demonstrate that our self-energy-corrected wave functions can be used for further calculations of one- and two-loop quantumelectrodynamic corrections, including heretofore uncalculated
TABLE II. Comparison of our values for $\Delta E_{\mathrm{LAL}}$ obtained according to Eq. (46) (first column) and the values from Refs. [16,17] (second column) for the ground-state $1 s$ electron. With an asterisk we mark the values obtained by an interpolation of the results presented in Refs. [16,17]. We write $\Delta E_{\mathrm{LAL}}=\left(\frac{\alpha}{\pi}\right)^{2}(Z \alpha)^{4} F(Z \alpha)$. For high $Z$, the difference between the results is caused by the finite nuclear size effect.

\begin{tabular}{lcc}
\hline \hline$Z$ & $F(Z \alpha)$, Eq. $(46)$ & $F(Z \alpha)$, earlier works \\
\hline 10 & $-0.36(3)$ & $-0.358[16]$ \\
18 & $-0.561(2)$ & $-0.562^{*}$ \\
36 & $-0.828(2)$ & $-0.827^{*}$ \\
54 & $-1.013(2)$ & $-1.016^{*}$ \\
92 & $-1.680(2)$ & $-1.734[17]$ \\
\hline
\end{tabular}

ones. The results of our calculations in coordinate and momentum representations are tabulated in the Supplemental Material [48] and can be used for various calculations.

\section{ACKNOWLEDGMENTS}

This paper comprises parts of the Ph.D. thesis work of H.C., to be submitted at the Heidelberg University, Germany. This work is part of and supported by the DFG Collaborative Research Centre SFB 1225 (ISOQUANT). V.A.Y. acknowledges support by the Ministry of Education and Science of the Russian Federation Grant No. 3.5397.2017/6.7.
[1] V. M. Shabaev, O. V. Andreev, A. N. Artemyev, S. S. Baturin, A. A. Elizarov, Y. S. Kozhedub, N. S. Oreshkina, I. I. Tupitsyn, V. A. Yerokhin, and O. M. Zherebtsov, Int. J. Mass Spectrom. 251, 109 (2006).

[2] V. M. Shabaev, D. A. Glazov, G. Plunien, and A. V. Volotka, J. Phys. Chem. Ref. Data 44, 031205 (2015).

[3] A. V. Volotka, D. A. Glazov, O. V. Andreev, V. M. Shabaev, I. I. Tupitsyn, and G. Plunien, Phys. Rev. Lett. 108, 073001 (2012).

[4] I. Arapoglou, A. Egl, M. Höcker, T. Sailer, B. Tu, A. Weigel, R. Wolf, H. Cakir, V. A. Yerokhin, N. S. Oreshkina, V. A. Agababaev, A. V. Volotka, D. V. Zinenko, D. A. Glazov, Z. Harman, C. H. Keitel, S. Sturm, and K. Blaum, Phys. Rev. Lett. 122, 253001 (2019).

[5] A. Egl, I. Arapoglou, M. Höcker, K. König, T. Ratajczyk, T. Sailer, B. Tu, A. Weigel, K. Blaum, W. Nörtershäuser, and S. Sturm, Phys. Rev. Lett. 123, 123001 (2019).

[6] S. Sturm, F. Köhler, J. Zatorski, A. Wagner, Z. Harman, G. Werth, W. Quint, C. H. Keitel, and K. Blaum, Nature (London) 506, 467 (2014).

[7] V. M. Shabaev, D. A. Glazov, N. S. Oreshkina, A. V. Volotka, G. Plunien, H.-J. Kluge, and W. Quint, Phys. Rev. Lett. 96, 253002 (2006).

[8] V. A. Yerokhin, E. Berseneva, Z. Harman, I. I. Tupitsyn, and C. H. Keitel, Phys. Rev. Lett. 116, 100801 (2016).

[9] A. V. Volotka and G. Plunien, Phys. Rev. Lett. 113, 023002 (2014).

[10] B. Sikora, H. Cakir, N. Michel, V. Debierre, N. S. Oreshkina, N. A. Belov, V. A. Yerokhin, C. H. Keitel, and Z. Harman, Phys. Rev. D 97, 111301(R) (2018).
[11] A. Windberger, J. R. Crespo López-Urrutia, H. Bekker, N. S. Oreshkina, J. C. Berengut, V. Bock, A. Borschevsky, V. A. Dzuba, E. Eliav, Z. Harman, U. Kaldor, S. Kaul, U. I. Safronova, V. V. Flambaum, C. H. Keitel, P. O. Schmidt, J. Ullrich, and O. O. Versolato, Phys. Rev. Lett. 114, 150801 (2015).

[12] O. Y. Andreev, L. N. Labzowsky, G. Plunien, and G. Soff, Phys. Rev. Lett. 94, 243002 (2005).

[13] N. S. Oreshkina, S. M. Cavaletto, N. Michel, Z. Harman, and C. H. Keitel, Phys. Rev. A 96, 030501(R) (2017).

[14] J. Holmberg, A. N. Artemyev, A. Surzhykov, V. A. Yerokhin, and T. Stöhlker, Phys. Rev. A 92, 042510 (2015).

[15] T. Beier, Phys. Rep. 339, 79 (2000).

[16] V. A. Yerokhin, Eur. Phys. J. D 58, 57 (2010).

[17] V. A. Yerokhin, Phys. Rev. A 97, 052509 (2018).

[18] A. M. Desiderio and W. R. Johnson, Phys. Rev. A 3, 1267 (1971).

[19] J. S. L. G. E. Brown and G. W. Schaefer, Proc. R. Soc. A 251 , 92 (1959).

[20] P. J. Mohr, Ann. Phys. (NY) 88, 26 (1974).

[21] P. J. Mohr, Ann. Phys. (NY) 88, 52 (1974).

[22] P. J. Mohr, Phys. Rev. A 26, 2338 (1982).

[23] P. Indelicato and P. J. Mohr, Phys. Rev. A 46, 172 (1992)

[24] P. J. Mohr, G. Plunien, and G. Soff, Phys. Rep. 293, 227 (1998).

[25] N. J. Snyderman, Ann. Phys. (NY) 211, 43 (1991).

[26] S. A. Blundell and N. J. Snyderman, Phys. Rev. A 44, R1427(R) (1991). 
[27] K. T. Cheng, W. R. Johnson, and J. Sapirstein, Phys. Rev. A 47, 1817 (1993).

[28] P. Sunnergren, H. Persson, S. Salomonson, S. M. Schneider, I. Lindgren, and G. Soff, Phys. Rev. A 58, 1055 (1998).

[29] V. A. Yerokhin and V. M. Shabaev, Phys. Rev. A 60, 800 (1999).

[30] W. Greiner, Relativistic Quantum Mechanics, 3rd ed. (SpringerVerlag, Berlin, 2000).

[31] V. A. Yerokhin, P. Indelicato, and V. M. Shabaev, Phys. Rev. A 69, 052503 (2004).

[32] K. Pachucki, A. Czarnecki, U. D. Jentschura, and V. A. Yerokhin, Phys. Rev. A 72, 022108 (2005).

[33] N. S. Oreshkina, D. A. Glazov, A. Volotka, V. M. Shabaev, I. I. Tupitsyn, and G. Plunien, Phys. Lett. A 372, 675 (2008).

[34] A. V. Volotka, D. A. Glazov, I. I. Tupitsyn, N. S. Oreshkina, G. Plunien, and V. M. Shabaev, Phys. Rev. A 78, 062507 (2008).

[35] V. A. Yerokhin, P. Indelicato, and V. M. Shabaev, Phys. Rev. Lett. 91, 073001 (2003).

[36] V. A. Yerokhin, P. Indelicato, and V. M. Shabaev, Phys. Rev. Lett. 97, 253004 (2006).

[37] S. Mallampalli and J. Sapirstein, Phys. Rev. A 57, 1548 (1998).

[38] S. Mallampalli and J. Sapirstein, Phys. Rev. Lett. 80, 5297 (1998).
[39] B. Sikora, V. A. Yerokhin, N. S. Oreshkina, H. Cakir, C. H. Keitel, and Z. Harman, Phys. Rev. Res. 2, 012002(R) (2020).

[40] V. Debierre, H. Cakir, B. Sikora, N. S. Oreshkina, Z. Harman, and C. H. Keitel (unpublished).

[41] S. A. Blundell, Phys. Rev. A 46, 3762 (1992).

[42] D. A. Varshalovich, A. N. Moskalev, and V. K. Khersonskii, Quantum Theory of Angular Momentum (World Scientific, Singapore, 1988).

[43] V. M. Shabaev, I. I. Tupitsyn, V. A. Yerokhin, G. Plunien, and G. Soff, Phys. Rev. Lett. 93, 130405 (2004).

[44] W. R. Johnson and J. Sapirstein, Phys. Rev. Lett. 57, 1126 (1986)

[45] W. R. Johnson, S. A. Blundell, and J. Sapirstein, Phys. Rev. A 37, 307 (1988).

[46] I. Angeli and K. P. Marinova, At. Data Nucl. Data Tables 99, 69 (2013).

[47] QUADPACK, http://www.netlib.org/quadpack/.

[48] See Supplemental Material at http://link.aps.org/supplemental/ 10.1103/PhysRevA.101.032511 for the tabulated SE-WFs in coordinate and momentum representations, calculated on two different grids for the ground $1 s$ state of hydrogenlike ions with $Z=2,10,18,36,54,92$. 